\title{
North American Prodrome Longitudinal Study (NAPLS 2): Overview and Recruitment
}

\author{
Jean Addington ${ }^{1}$, Kristin S. Cadenhead ${ }^{2}$, Barbara A. Cornblatt ${ }^{3}$, Daniel H. Mathalon ${ }^{4}$, \\ Thomas H. McGlashan ${ }^{5}$, Diana O. Perkins ${ }^{6}$, Larry J. Seidman ${ }^{7}$, Ming T. Tsuang ${ }^{3}$, Elaine F. \\ Walker $^{8}$, Scott W. Woods ${ }^{4}$, Jack A. Addington ${ }^{1}$, and Tyrone D. Cannon ${ }^{9}$ \\ ${ }^{1}$ Department of Psychiatry, University of Calgary, Calgary, Alberta, Canada \\ ${ }^{2}$ Department of Psychiatry, UCSD, San Diego CA \\ ${ }^{3}$ Department of Psychiatry, Zucker Hillside Hospital, Long Island NY \\ 4Department of Psychiatry, UCSF, and SFVA Medical Center, San Francisco CA \\ ${ }^{5}$ Department of Psychiatry, Yale University, New Haven CT \\ ${ }^{6}$ Department of Psychiatry, University of North Carolina, Chapel Hill NC \\ ${ }^{7}$ Department of Psychiatry, Harvard Medical School at Beth Israel Deaconess Medical Center and \\ Massachusetts General Hospital, Boston MA \\ ${ }^{8}$ Departments of Psychology and Psychiatry, Emory University, Atlanta GA \\ ${ }^{9}$ Departments of Psychology and Psychiatry and Biobehavioral Sciences, UCLA, Los Angeles CA
}

\section{Abstract}

The North American Prodrome Longitudinal Study (NAPLS) is a consortium of eight programs focusing on the psychosis prodrome. Funded by the National Institute of Mental Health (NIMH), the sites are located at Emory University, Harvard University, University of Calgary, UCLA, UCSD, University of North Carolina Chapel Hill, Yale University, and Zucker Hillside Hospital. Although the programs initially developed independently, they previously collaborated to combine their historical datasets and to produce a series of analyses on predictors of psychosis in one of the largest samples of longitudinally followed prodromal subjects worldwide. This led to the development of a five year prospective study "Predictors and Mechanisms of Conversion to Psychosis", (also known as NAPLS-2) with three major aims: (1) to prospectively test the prediction algorithm developed in NAPLS-1, (2) to investigate the neuroanatomical, neurophysiological, neurocognitive, and neurohormonal factors that may contribute to the development of psychosis, and (3) to develop a repository of DNA, RNA, and plasma from

\footnotetext{
(C) 2012 Elsevier B.V. All rights reserved.

"Corresponding Author: Dr Jean Addington, Mathison Centre for Mental Health Research and Education, University of Calgary, 3280 Hospital Drive NW, Calgary, Alberta T2N 4Z6 Canada. jmadding@ucalgary.ca.

Contributors

All authors were responsible for the design of the whole study or aspects of it and the supervision of all aspects of data collection. Dr Jean Addington took the lead on writing the manuscript with help in writing from all authors. All authors contributed to and approved the final manuscript.

Conflict of interest

There are no conflicts of interest for any of the authors with respect to the data in this paper or for the study.

Publisher's Disclaimer: This is a PDF file of an unedited manuscript that has been accepted for publication. As a service to our customers we are providing this early version of the manuscript. The manuscript will undergo copyediting, typesetting, and review of the resulting proof before it is published in its final citable form. Please note that during the production process errors may be discovered which could affect the content, and all legal disclaimers that apply to the journal pertain.
} 
participants meeting diagnostic criteria for a clinical high risk (CHR) state and from demographically similar healthy subjects. Funded by NIMH in 2008, NAPLS-2 will generate the largest CHR for psychosis sample with 720 CHR and 240 healthy comparison subjects, and thus will provide statistical power and scientific scope that cannot be duplicated by any single site study. This paper describes the overall methodology of the NAPLS-2 project and reports on the ascertainment and demographics at the midway point of the study with (360 CHR) and 180 controls.

\section{INTRODUCTION}

Interest in early detection and prevention of schizophrenia and other psychotic disorders stems from evidence of brain structural changes and decline in function around the time of psychosis onset, suggesting that the pre-onset or 'prodromal' period affords a window of opportunity for preventive intervention. Identifying predictors and mechanisms of conversion to psychosis among such individuals ascertained to be in a clinical high risk (CHR) or prodromal clinical state are critical steps in the search for preventive strategies. Achieving these aims requires sample sizes much larger than those typically available at a single research centre within a reasonable time period.

The North American Prodrome Longitudinal Study (NAPLS) is a consortium of eight programs focusing on the psychosis prodrome. The sites are located at Emory University, Harvard University, University of Calgary in Canada, University of California at Los Angeles, University of California at San Diego, University of North Carolina at Chapel Hill, Yale University, and Zucker Hillside Hospital. In a prior phase of the project, these sites collaborated to combine previously collected datasets and produced a series of analyses on predictors of psychosis in the largest sample of longitudinally followed prodromal subjects worldwide (N=291) (Addington et al., 2007). Results of these analyses indicated that risk for the onset of psychosis in this population was $35 \%$ after $2 \frac{1}{2}$ years of follow-up, with a decelerating rate of conversion over this period. The NAPLS data set was used to derive a psychosis prediction algorithm with high positive predictive power ( $\sim 80 \%)$, but only modest sensitivity ( 40\%).(Cannon et al., 2008) The published prediction algorithm included genetic risk (having a first degree relative with a psychotic disorder and functional decline), more severe unusual thought content, and greater social impairment.

These preliminary efforts led to a five year prospective study "Predictors and Mechanisms of Conversion to Psychosis", funded by NIMH in 2008, also referred to as NAPLS 2 that included all eight NAPLS sites. The number of subjects in NAPLS 2 is anticipated to be sufficient to address fundamental questions about the neurobiological correlates of the development of psychosis. The goal of recruiting a sample of 720 clinical high risk participants and 240 healthy controls will be achieved by the end of 2012, and based on the previously collected NAPLS 1 data, a conversion rate of approximately $30 \%$ is expected with up to two year follow-up.

This paper describes the overall methodology of the NAPLS 2 project and reports on the ascertainment and demographics of the first half of the CHR sample $(\mathrm{N}=360)$ and 180 of the healthy controls.

\section{AIMS OF THE PROJECT}

The central pathophysiologic assumption underlying the project is that schizophrenia (and other psychotic disorders) reflect a process of brain volume reduction involving pruning of synapses and dendrites, that result in reduced cortical connectivity (McGlashan and Hoffman, 2000), particularly in prefrontal and superior and medial temporal lobe regions 
governing attention, executive, auditory-language, and memory-related functions. The first project aim is to determine whether the NAPLS 1 psychosis risk prediction algorithm can be replicated in a new and larger sample of CHR individuals. The hypothesis is that each of four factors: genetic vulnerability to schizophrenia with recent deterioration in functioning, higher levels of unusual thought content, higher levels of suspicion-paranoia, greater social impairment at baseline, will independently predict a higher risk for conversion to psychosis, and that combining all of these indicators will substantially reduce false positive psychosis predictions over two years of follow-up. The second is to determine whether biological and neurocognitive abnormalities preceding psychosis onset contribute to predicting psychosis independently of the clinical algorithms and whether they can be combined with the clinical measures to enhance predictive utility.

The third aim is to determine whether neuroanatomical, neurophysiological, neurocognitive, and neurohormonal abnormalities that precede psychosis represent stable vulnerability markers or markers of progression during the prodromal phase. This will enable us to determine whether CHR individuals who convert to psychosis show a steeper rate of change in neurobiological risk indicators compared to non-converters and healthy controls. The final aim is to develop a repository of DNA and RNA from participants meeting diagnostic criteria for a CHR state and from demographically similar healthy participants.

\section{NAPLS TASK FORCES}

In NAPLS each site contributes an area of uniqueness to the domain of assessment. The Clinical Task Force is led by J. Addington (Calgary) and consists of psychodiagnostics (JA $\& \mathrm{TMcG}$ ), psychopathology (JA) and early risk factors (JA). The Social Task Force is led by B. Cornblatt (Zucker Hillside Hospital (ZHH)) with a focus on social and role functioning (BC) and social cognition (JA). Neuropsychology is directed by L Seidman (Harvard). Treatment is monitored by S. Woods (Yale). Neuroimaging is directed by T. Cannon (UCLA). The electrophysiology Task Force is co-chaired by K. Cadenhead (UCSD) and D. Mathalon (UCSF). E. Walker (Emory) is the chair of the Stress Hormone Task Force. D. Perkins (UNC) and M. Tsuang (UCSD) co-chair the Genomics Task Force.

The task force chair(s) were responsible for developing and coordinating each aspect of the study protocol including ongoing training and quality assurance procedures in all assessments. Rigorous protocols for training, standardization of procedures and maintenance of reliability have been applied across all sites by each task force. Following the initial startup, annual meetings to monitor quality assurance have been convened.

\section{ASSESSMENTS}

The clinical component of the project attempts to replicate and refine the major NAPLS 1 prediction findings. The biomarkers component tests potential mechanisms of illness onset and/or progression in the neuroanatomical, electrophysiological, neurohormonal, neuropsychological, and genomic domains. Selection of measures attempted to reflect both vulnerability-related and progressive neuromaturational processes. Selection was limited to measures for which evidence existed with relevance to the psychosis prodrome, and which were likely to reflect pathophysiologic changes associated with clinical and/or functional deterioration.

We required proven methods for the reliable collection and aggregation of data across measurement points and across sites. The selected measures span multiple critical levels of analysis (i.e. genomic, hormonal, anatomical, physiological and behavioral). Such a multilevel perspective was assumed to be necessary given that the aberrant molecular and cellular 
processes underlying psychotic disorders were likely to reflect cascading influences across these five critical domains.

Baseline only measures include demographics, premorbid functioning, life events and childhood trauma. Six monthly clinical assessments include SIPS, anxiety, depression, substance use and social functioning. Logs of medications, psychosocial treatments and resource utilization are updated every 6 months. Biomarker assessments of neurocognition, electrophysiology, cortisol, blood draws, and imaging occur at baseline, 1 and 2 years. Should a participant convert to psychosis, then the complete assessment is done at the time of conversion with a one year post conversion assessment to determine the diagnosis.

\section{ASCERTAINMENT}

NAPLS participants are help-seeking. They are referred from health care providers, educators, or social service agencies or they self-refer in response to intensive community education efforts. These initiatives included academic detailing, grand rounds, educational talks, mailings, postings, websites and internet hits, and public service announcements. Each site has developed extensive referral sources in their area, and routinely contact them personally, with mail outs, and through educational efforts. Potential participants undergo a telephone screen. Those who screen positive are invited to an in-person eligibility and consent evaluation.

The first half of the sample $\mathrm{N}=360$ was recruited over a 2 year period from January 2009 until January 2011. Overall, 1749 referrals were received as depicted in Figure 1. Based on a phone screen 618 clearly did not meet CHR criteria, but $52 \%$ were found to be suitable for a screening interview. Of those deemed not suitable at screening some were psychotic. Examples of other reasons included not having prodromal symptoms, calling about other problems such as anxiety, depression, severe autism, non-English speaking, or outside the age range. Of those offered screening, 28\% did not keep their scheduled interview and 28\% were found not to meet CHR criteria. Examples of reasons for not meeting criteria at the assessment included prodromal symptoms being longstanding, not frequent enough or no longer present. Outcome of all referrals are presented in Figure 1. There were some site differences. Yale and ZHH received significantly more referrals and ruled out more at phone screening. UNC had a higher proportion of referrals that they were unable to contact further. San Diego, Emory and Yale had a higher proportion of no shows.

Figure 2 depicts the referral sources and the number meeting prodromal criteria from each source. Overall, significantly more referrals came from self and family, although the majority of such referrals did not meet criteria. UCLA, Harvard, UCSD and Calgary recruited from a wide range of clinical and community services. Although Yale did receive some referrals from clinical services the majority of Yale's were self-referrals. Referrals for ZHH were made primarily by the outpatient department intake staff and other clinical services that informed parents to contact the program directly. The majority of the referrals at Emory and UNC were self-referrals, followed by family and friends.

Figure 3 elaborates on the marketing strategies and their effectiveness. Presentations and web and internet searches seemed to be the most successful, as was "word of mouth". This was the case at most of the sites; the one exception being Emory where the majority of the referrals resulted from advertising in public places.

\section{SAMPLE}

The clinical high risk sample met the Criteria of Prodromal Syndromes (COPS) which is based on the Structured Interview for Prodromal Syndromes (SIPS).(McGlashan et al., 
2010) After a comprehensive assessment that includes administering the Structure Clinical Interview for DSM-IV (SCID) (First et al., 1995) and the SIPS, vignettes are developed for each CHR participant for the purpose of obtaining a consensus diagnosis. The attenuated psychotic symptoms rated on the Scale of Prodromal Symptoms (SOPS) are described at length and include both recent and longstanding symptoms. The vignette is written so that another rater can review the information under each symptom category and provide a reliable rating. Once approved at the site level, the vignette is presented on a conference call for a consensus decision on the symptom ratings as well as the diagnosis. The NAPLS-2 consensus call, chaired by JA, is held once a week and is attended by members of each of the eight study sites including the authors of the SIPS, Drs McGlashan and Walsh, who act as co-chairs. Submitted vignettes are individually reviewed and a consensus must be reached on each symptom rating, diagnosis and ultimate admission into the study. It is often challenging making differentiations with respect to some of the exclusion criteria listed below. But we use our calls to discuss issues such as the impact of substance abuse and use of antipsychotics. Such decisions can later be reviewed when we have followed the sample and determined the outcome of the participants in terms of conversion and remission.

Cross-site reliability in the ratings of the SOPS, was conducted on a yearly basis using a new videotape each year. Ratings from all raters at all sites were compared to "gold standard" ratings on the SOPS. Intra class correlations, over three years, for the total SOPS scores ranged from 0.82 to 0.93 and for the attenuated positive symptom score from the SOPS ranged from $0.92-0.96$. There were minimal differences across the individual sites. All interclass correlations were in the excellent range.

\subsection{Inclusion and exclusion criteria}

Those at CHR had to be between 12 and 35 years old and meet diagnostic criteria for a prodromal syndrome as per the COPS criteria (McGlashan et al., 2010) or if under 19 meet criteria for schizotypal personality disorder (SPD). Participants were excluded if they met criteria for current or lifetime Axis I psychotic disorder, including affective psychoses, IQ > 70 , or had a past history of a central nervous system disorder, or substance dependence in the past 6 months or the diagnostic prodromal symptoms were clearly caused by an Axis 1 disorder. Other non-psychotic DSM-IV disorders were not exclusionary (e.g. substance abuse disorder, major depression, anxiety disorders, Axis II disorders), as long as the disorder did not account for the individual's prodromal symptoms. Use of antipsychotics was not an exclusion provided there was clear evidence that prodromal but not psychotic symptoms were present when the medication was started. Control subjects could not meet criteria for any prodromal syndrome, any current or past psychotic disorder or a Cluster A personality disorder diagnosis, not have a family history (in first-degree relatives) of any psychotic disorder or any other disorder involving psychotic symptoms. They could not be currently using psychotropic medication.

\subsection{Demographics}

The samples' age ranges from 12-34 years with a mean (SD) age of 18.99 (4.18) for CHR and 19.54 (4.77) for healthy controls (HC). There are significantly more males in the CHR group $\left(211,58.6 \%\right.$ vs $\left.87,48.3 \%, \chi^{2}=5.12, \mathrm{p}<0.05\right)$. The groups did not differ in ethnicity with the majority being white (CHR 55\%, HC 59\%) or marital status with the majority (85\%) being single or living at home (CHR 75\%, HC 63\%) or enrolled as a student (CHR $80.3 \%$, HC $82.2 \%$ ). Significantly more of the controls were working ( $46 \%$ vs $28 \%, \chi^{2}=$ $13.97, \mathrm{p}<0.05)$ and had significantly more years of education $(12.39, \mathrm{SD}=3.60$ vs $11: 56$ $\mathrm{SD}=2.63, \mathrm{t}=3.09, \mathrm{p}<0.01$ ). This may reflect the clinical nature of the sample. In addition the majority of all participants were born in the USA or Canada. Eighty percent of CHR and 93\% of control participants had English as their first language. Spanish was the first 
language of 50\% of the non-English speaking group. We attempted to frequency match the groups on age and gender.

\subsection{Clinical presentation}

The CHR participants are a help-seeking sample and as such present not only with subthreshold symptoms but also a wide range of comorbidity. The majority (80\%) meet criteria for the attenuated positive symptoms syndrome with a small proportion meeting another criteria or a combination of criteria. These CHR subtype diagnoses are presented in Table 1. Table 2 reports on the diagnoses that differentiated the CHR from the control group. Although the substance diagnoses for cannabis in particular appear low these are individuals that meet diagnoses for abuse and/or dependence. For cannabis approximately $30 \%$ of the controls and $40 \%$ of the CHR rated themselves as current users.

\subsection{Site differences}

We compared the demographics across sites to assess heterogeneity. There were differences for the CHR in age, years of education and gender. ZHH followed by UCLA had the youngest participants with Emory having the oldest. ZHH had significantly younger participants than Emory, UNC, San Diego and Yale and UCLA had significantly younger participants than Emory. Related to age, participants at ZHH had significantly fewer years of education than participants from Emory, UNC and UCSD. UCLA participants had fewer years of education compared to UNC. Harvard, Emory and UNC had equal numbers of males to females. Ratios of males to females at the other sites ranged from 70:30 to 62:38.

With respect to racial background, Emory had significantly more African American participants than any other site at 50\% with UCSD and Calgary having the least. Most sites had $40-60 \%$ white participants with there being $65 \%$ at UNC and $75 \%$ at Calgary.

\section{DATA MANAGEMENT}

One concern with large multisite, longitudinal studies including many assessments is data integrity. Data are managed at the Calgary site through a centralized Oracle database. Sites can remotely manage both the input of participant study data and also track participant protocol adherence and staff task management. Data input is strictly controlled with data validation prior to saving and a two-step forced resolution of required data. No data can be submitted until missing data fields are flagged with either reasons for absence or with a predetermined code. Prior to forcing a second blind double entry of all the data, all missing data reasons must be validated and signed off with an acceptance code by a data manager from the site. This rigorous feedback loop ensures better data collection, near perfect data entry and much better data quality assurance. These codes can then be reported and reviewed by the appropriate Task Force to determine further action. The system produces numerous reports. These are used to track recruitment, dropout rates, data collection and consequent data entry completion levels. Furthermore, it can measure the utility and validity of measures and the protocol. This allows the data cleanup process to be continuous. There is an overall data manager at the Calgary site with each site having their own data manager to work with both within and across site data.

\section{DISCUSSION}

NAPLS-2 will be the one of the largest cohort studies of young people at clinical high risk for psychosis to be followed longitudinally. It will be the first to study all of these clinical and biological factors prospectively and simultaneously in a large and well-characterized sample. This paper has described the aims and methods of the project and reported on recruitment and preliminary descriptive data from the first half of the sample. The 
recruitment practices are similar across most of the sites. There are very few significant site differences in samples and no-one site differs on more than one variable. Yet one of the advantages of having multiple sites is that they are diverse with respect to the regions and ethnicities represented. Our multi-site approach affords the opportunity to examine regional and ethnic differences in the ascertainment of CHR individuals, as well as the nature and course of prodromal syndromes. Further, by having multiple sites, we are able to validate our measures across a range of clinical research facilities, and this has important benefits with respect to establishing the generalizability of NAPLS findings, especially those that bear on prediction algorithms Future reports will focus on baseline clinical and biological characteristics, longitudinal changes and eventually predictors of conversion.

\section{Acknowledgments}

\section{Acknowledgements to the NAPLS group}

J Stowkowy, T Raedler, L McGregor, D Marulanda, L Legere, L Liu, C Marshall, E Falukozi, E Fitton, K Smith (University of Calgary). T Alderman, K Shafer, I Domingues, A Hurria, H Mirzakhanian (UCSD). B Walsh, J Saksa, N Santamauro, A Carlson, J Kenney, B Roman (Yale University). K Woodberry, AJ Giuliano, W Stone, JM Rodenhiser, L Tucker, R Serur, G Min, R Szent-Imrey (Beth Israel Deaconess Medical Center/Harvard). C Bearden, P Bachman, J Zinberg, S DeSilva, A Andaya, S Uguryan (UCLA). J Brasfield, H Trotman, (Emory University). A Pelletier, K Lansing, H Mates, J Nieri, B Landaas, K Graham, E Rothman, J Hurta, Y Sierra (University of North Carolina). A Auther, R Carrion, M McLaughlin, R Olsen (Zucker Hillside Hospital)

\section{Role of funding source}

This study was supported by the National Institute of Mental Health (grant U01MH081984 to Dr Addington; grants U01 MH081928; P50 MH080272; Commonwealth of Massachusetts SCDMH82101008006 to Dr Seidman; grants R01 MH60720, U01 MH082022 and K24 MH76191 to Dr Cadenhead; grant to Dr Cannon; grant U01MH082004-01A1 to Dr Perkins; grant U01MH081988 to Dr Walker; grant U01MH082022 to Dr Woods; and UO1 MH081857-05 grant to Dr Cornblatt. The NIMH had no further role in study design; in the collection, analysis and interpretation of data; in the writing of the report; and in the decision to submit the paper for publication.

\section{Reference List}

Addington J, Cadenhead KS, Cannon TD, Cornblatt B, McGlashan TH, Perkins DO, Seidman LJ, Tsuang M, Walker EF, Woods SW, Heinssen R. North American Prodrome Longitudinal Study: A Collaborative Multisite Approach to Prodromal Schizophrenia Research. Schizophr Bull. 2007

Cannon TD, Cadenhead K, Cornblatt B, Woods SW, Addington J, Walker E, Seidman LJ, Perkins D, Tsuang M, McGlashan T, Heinssen R. Prediction of psychosis in youth at high clinical risk: a multisite longitudinal study in North America. Arch Gen Psychiatry. 2008; 65:28-37. [PubMed: 18180426]

First, M.; Spitzer, RL.; Gibbon, M.; Williams, B.; Williams, JBW. Structured Clinical Interview for DSM-IV Axis I Disorders, Patient Edition. Biometrics Research Department, New York State Psychiatric Institute, New York; New York: 1995.

McGlashan, T.; Walsh, BC.; Woods, SW. The Psychosis Risk Syndrome: Handbook for Diagnosis and Follow-up. Oxford University Press; New York, New York: 2010.

McGlashan TH, Hoffman RE. Schizophrenia as a Disorder of Developmentally Reduced Synaptic Connectivity. Arch Gen Psychiatry. 2000; 57:637-648. [PubMed: 10891034] 


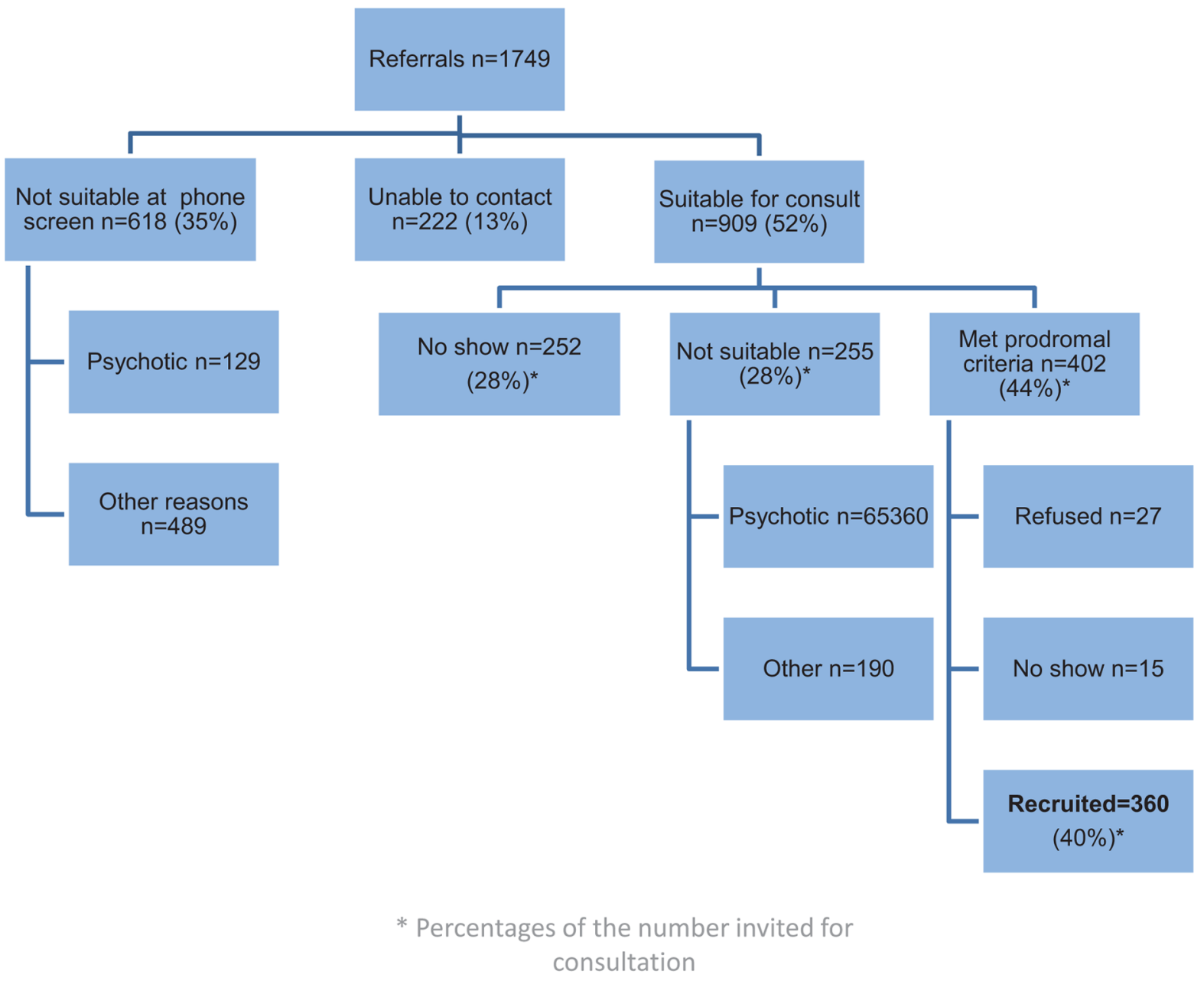

Figure 1.

Outcome of All Referrals to NAPLS 


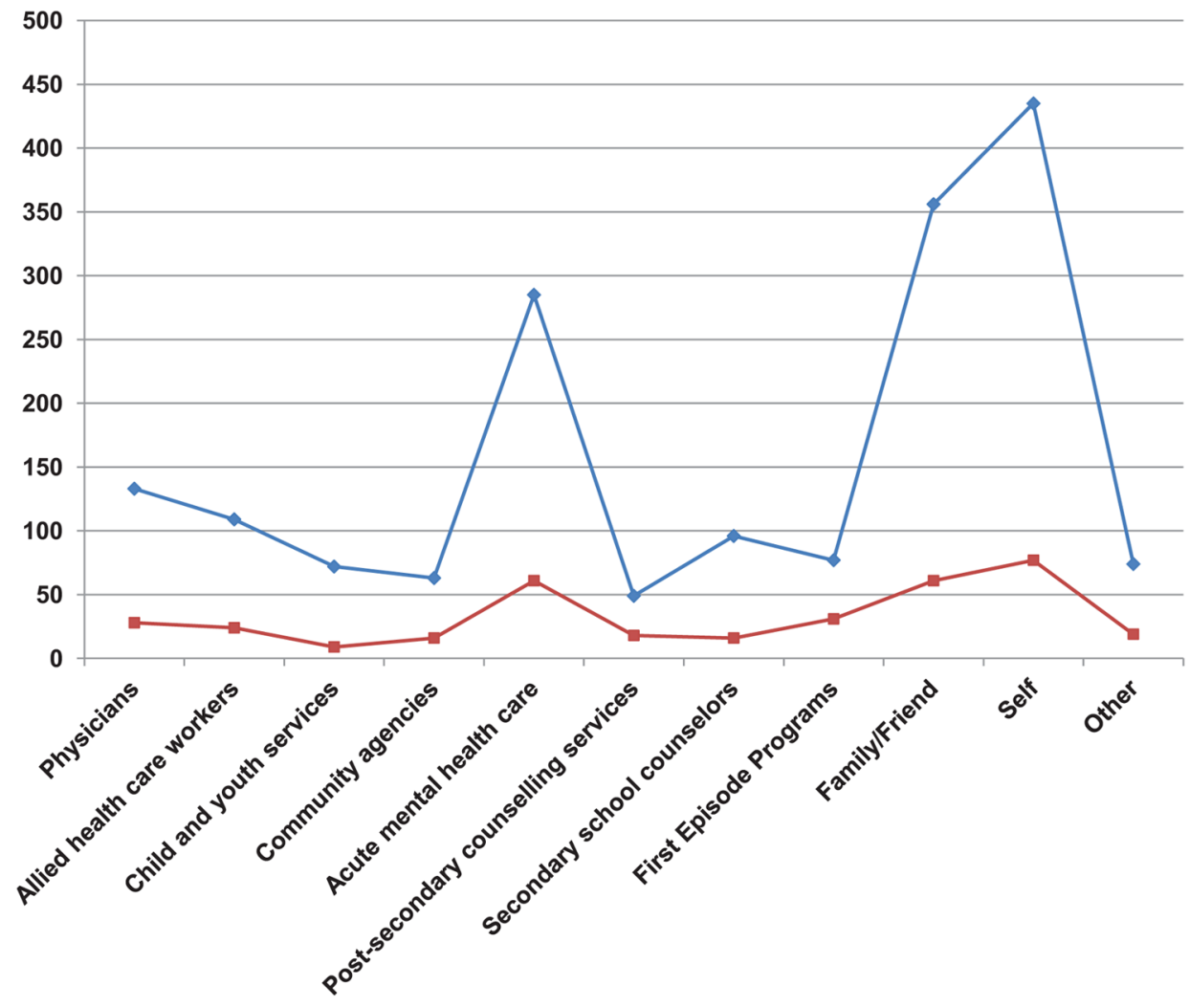

Figure 2.

Referral Sources 


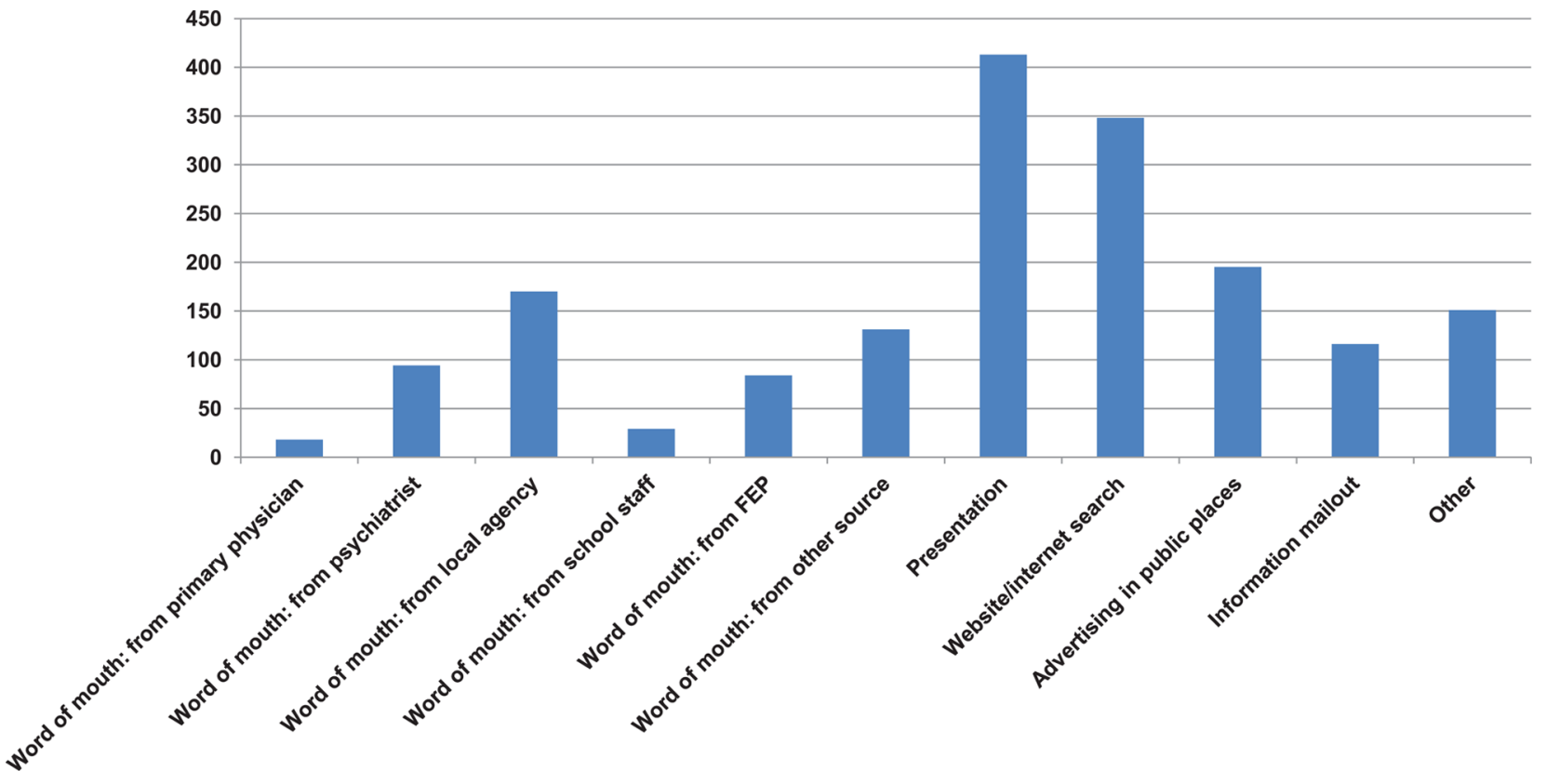

Figure 3.

Marketing 
Table 1

Prodromal Diagnostic Criteria

\begin{tabular}{|l|l|}
\hline Criteria & Percent of total \\
\hline Attenuated positive symptoms (APS) & 80.2 \\
\hline Genetic risk and deterioration (GRD) & 4.7 \\
\hline Brief Intermittent positive symptoms (BIPS) & 0.4 \\
\hline APS \& GRD & 9.3 \\
\hline APS \& BIPS & 1.9 \\
\hline BIPS \& GRD & 0 \\
\hline APS \& GRD \& BIPS & 0 \\
\hline Under 19 plus schizotypy & 4.4 \\
\hline
\end{tabular}


Table 2

SCID diagnoses

\begin{tabular}{|c|c|c|c|}
\hline SCID-IV Diagnosis & Clinical High Risk (N=360) & Control (N=108) & Significance of $\chi^{2}$ \\
\hline Depression- Current & $41.4 \%$ & $1.7 \%$ & $<0.0001$ \\
\hline Depression- Lifetime & $35.1 \%$ & $5.6 \%$ & $<0.0001$ \\
\hline Lifetime Alcohol & $10.7 \%$ & $1.1 \%$ & $<0.0001$ \\
\hline Current Cannabis & $5.8 \%$ & $1.1 \%$ & $<0.05$ \\
\hline Lifetime Cannabis & $12.8 \%$ & $2.8 \%$ & $<0.001$ \\
\hline Current OCD & $7.8 \%$ & $0 \%$ & $<0.0001$ \\
\hline Lifetime OCD & $4.6 \%$ & $0 \%$ & $<0.01$ \\
\hline Current PTSD & $2.6 \%$ & $0 \%$ & $<0.05$ \\
\hline Lifetime PTSD & $4.1 \%$ & $0 \%$ & $<0.01$ \\
\hline Current Panic & $13.3 \%$ & $0.6 \%$ & $<0.0001$ \\
\hline Lifetime Panic & $9.3 \%$ & $0 \%$ & $<0.0001$ \\
\hline Current Social Phobia & $13.6 \%$ & $0 \%$ & $<0.0001$ \\
\hline Lifetime Social Phobia & $7.5 \%$ & $0.6 \%$ & $<0.0001$ \\
\hline Current Specific Phobia & $11.0 \%$ & $0 \%$ & $<0.0001$ \\
\hline Lifetime Specific Phobia & $6.4 \%$ & $0.6 \%$ & $<0.01$ \\
\hline Current GAD or NOS & $20.3 \%$ & $2.2 \%$ & $<0.0001$ \\
\hline Lifetime GAD or NOS & $9.0 \%$ & $0.6 \%$ & $<0.001$ \\
\hline Current Attention- Deficit/Hyperactivity & $15.7 \%$ & $1.7 \%$ & $<0.0001$ \\
\hline Lifetime Attention- Deficit/Hyperactivity & $10.8 \%$ & $2.2 \%$ & $<0.01$ \\
\hline Current Learning Disorder NOS & $2.3 \%$ & $0 \%$ & $<0.05$ \\
\hline Current Avoidant & $10.4 \%$ & $0 \%$ & $<0.0001$ \\
\hline Lifetime Avoidant & $4.6 \%$ & $0 \%$ & $<0.01$ \\
\hline Current Schizotypal & $18.3 \%$ & $0 \%$ & $<0.0001$ \\
\hline Lifetime Schizotypal & $7.2 \%$ & $0 \%$ & $<0.0001$ \\
\hline Current Borderline & $3.8 \%$ & $0 \%$ & $<0.01$ \\
\hline
\end{tabular}

